\title{
Pulmonary emphysema is associated with fungal sensitization in asthma
}

\author{
Yuta Kono, Masako To, Ryuta Tsuzuki, Satoshi Yamawaki, Seiko Soeda, Yasuo To \\ Department of Allergy and Respiratory Medicine, The Fraternity Memorial Hospital, Tokyo, Japan \\ Contributions: (I) Conception and design: Y Kono; (II) Administrative support: None; (III) Provision of study materials or patients: None; (IV) \\ Collection and assembly of data: R Tsuzuki, S Yamawaki, S Soeda, Y To; (V) Data analysis and interpretation: M To, Y To; (VI) Manuscript writing: \\ All authors; (VII) Final approval of manuscript: All authors. \\ Correspondence to: Yuta Kono, MD, PhD. Department of Allergy and Respiratory Medicine, The Fraternity Memorial Hospital 2-1-11 Yokoami, \\ Sumida-ku, Tokyo, Japan. Email: yuta.kono1978@gmail.com.
}

\begin{abstract}
Background: Fungal sensitization is a risk factor for severe asthma. Colonization in the lower respiratory tract is one of the forms of fungal exposure, and it is related to fungal sensitization. Pulmonary emphysema was recently reported to be an underlying disease of fungal colonization. The aim of study was to evaluate the prevalence of pulmonary emphysema in asthmatic patients with and without fungal sensitization.

Methods: We enrolled 108 patients with allergic asthma and divided them into the patients sensitized to Aspergillus and/or Candida $(n=56)$ and those not sensitized to both Aspergillus and Candida $(n=52)$. The presence of pulmonary emphysema on chest CT was evaluated retrospectively.

Results: The frequency of pulmonary emphysema was significantly higher in the patients sensitized to Aspergillus and/or Candida compared to the patients not sensitized to both fungi $(\mathrm{P}=0.0040)$. The frequency of pulmonary emphysema was also significantly higher in the patients sensitized to either Aspergillus or Candida compared to the patient not sensitized to the fungi ( $\mathrm{P}=0.0398$ and $\mathrm{P}=0.0198$, respectively). A multivariate logistic regression analysis demonstrated that the presence of pulmonary emphysema was independently associated with the sensitization to Aspergillus and/or Candida (OR 7.84, 95\% CI: $1.20-$ 51.10).

Conclusions: Pulmonary emphysema is associated with sensitization to Aspergillus and/or Candida.
\end{abstract}

Keywords: Asthma; Aspergillus; Candida; emphysema; fungal sensitization

Submitted Feb 23, 2020. Accepted for publication Aug 13, 2020.

doi: $10.21037 /$ jtd-20-995

View this article at: http://dx.doi.org/10.21037/jtd-20-995

\section{Introduction}

Fungal sensitization is a risk factor for severe asthma (1-3). Accumulating evidence of the strong relationship between fungal sensitization and severe asthma led to the recognition of a new phenotype of severe asthma called severe asthma with fungal sensitization (SAFS) (3). According to a study that evaluated the characteristics of SAFS, $29 \%$ of patients with severe asthma had sensitization to at least one fungal allergen, and the most common fungal species were Candida (16\%), Aspergillus (11\%), and Trichophyton (11\%) (4). These fungi are well known to be related to severe asthma.

There are three forms of exposure to fungal allergens that are associated with severe asthma: (I) fungal colonization of the lungs, (II) inhalation of fungal allergens on spores or hyphae, and (III) fungal infection outside the respiratory tract, such as dermatophytes infection (5). Of these three forms of fungal exposure, the colonization of the lungs is thought to be involved in the sensitization to Aspergillus in bronchiectasis. Patients with asthma sensitized to Aspergillus have been reported to frequently have bronchiectasis (6). Moreover, Aspergillus has been known to often colonize lower respiratory tracts in patients 
with bronchiectasis (7). The colonization of Aspergillus caused by a disruption of local defense systems is thought to be associated with the sensitization to Aspergillus in bronchiectasis.

There are several diseases other than bronchiectasis that can make it easy for fungi to colonize the lower respiratory tract. In an investigation of underlying diseases of chronic pulmonary aspergillosis (including pulmonary aspergilloma which usually results from an ingrowth of colonized Aspergillus) (8), chronic obstructive pulmonary disease (COPD) and/or emphysema was shown to be a common pulmonary disease (9). Indeed, it is not rare to see patients who develop aspergilloma located in emphysematous bulla complicated with pulmonary emphysema. Moreover, in a study of the airway colonization of severe pulmonary emphysema in patients undergoing endoscopic lung volume reduction, colonizations of Candida, Aspergillus, and Penicillium were observed in the lower respiratory tract (10).

Given the relationship between fungal colonization and sensitization, pulmonary emphysema may have some associations with fungal sensitization. However, there are no reports addressing the relationship between pulmonary emphysema and fungal sensitization. Clinically, it is important to identify risk factors for fungal sensitization in asthma. We conducted the present study to clarify the association between pulmonary emphysema and sensitization to Aspergillus and Candida, which have been reported to be colonized in the lower respiratory tract of patients with pulmonary emphysema.

\section{Methods}

\section{Patients}

Patients with allergic asthma whose sensitization to Aspergillus and/or Candida were evaluated from April 2016 to July 2018 in The Fraternity Memorial Hospital were enrolled in this study. The inclusion criteria were as follows: (I) patients who were diagnosed with asthma based on the Global Initiative for Asthma (GINA) guidelines; (II) patients who showed positive radioallergosorbent test (RAST) results to environmental allergens related to asthmatic symptoms; (III) patients who underwent chest CT within 1 year before and after their sensitization to Aspergillus and/or Candida was evaluated; (IV) patients in whom we could determine whether or not there was a complicating asthma-COPD overlap (ACO). The sensitizations were evaluated by RAST. A positive RAST is defined as class 1 or greater. ACO was defined as patients who were $\geq 40$ years old and had a smoking history of $\geq 10$ pack-years and an $\mathrm{FEV}_{1} / \mathrm{FVC}$ of $<70 \%$. This study was conducted under the approval of the Ethics Committee of The Fraternity Memorial Hospital and in accordance with the Declaration of Helsinki (as revised in 2013). Individual consent for this retrospective analysis was waived.

\section{Study design}

This study was a retrospective analysis of patient cases. The prevalence of pulmonary emphysema in asthmatic patients with and without fungal sensitization were evaluated. We divided the enrolled patients into two groups: the patients with fungal asthma who showed sensitization to Aspergillus and/or Candida, and the patients with non-fungal asthma who did not show sensitization to either fungi. We analyzed the proportion of patients with pulmonary emphysema detected by chest CT in each group.

\section{CT imaging}

The presence of pulmonary emphysema was determined based on the presence of focal areas of low attenuation without visible walls. We also categorized emphysema into three types: centrilobular, panlobular, and paraseptal emphysema. A typical image of each type of emphysema is shown in Figure 1. The presence of bronchiectasis was determined based on the presence of one or more following features: (I) an internal diameter of the bronchus greater than that of the adjacent pulmonary artery, (II) a lack of tapering of the bronchial lumen toward the periphery, or (III) visualization of the bronchus within $10 \mathrm{~mm}$ of the pleura. The images were analyzed in consensus by two experienced pulmonologists (Y Kono, R Tsuzuki) blinded to clinical data including fungal sensitization.

\section{Statistical analyses}

$\mathrm{P}$ values $<0.05$ were considered significant. Categorical variables were compared by Fisher's exact test. The MannWhitney U-test was performed to analyze the differences between the groups. A multivariate logistic regression analysis was performed using EZR (Saitama Medical Center, Jichi Medical University, Saitama, Japan). Sensitization 

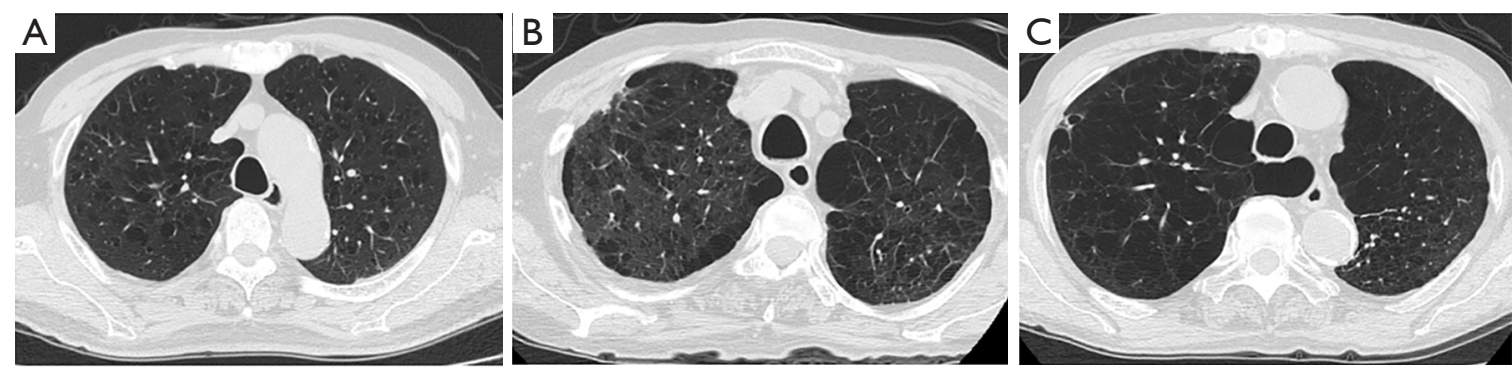

Figure 1 Typical CT images of pulmonary emphysema. (A) Centrilobular emphysema; (B) paraseptal emphysema; (C) panlobular emphysema.

to Aspergillus and/or Candida was used as a dependent variable, whereas age, gender, pack-years, and ACO were used as independent variables. All other statistical analyses were performed using the GraphPad Prism software program (GraphPad, San Diego, CA).

\section{Results}

\section{The study patients' characteristics}

We enrolled 108 patients with allergic asthma: 56 patients were categorized as having fungal asthma with sensitization to Aspergillus and/or Candida (the FA group), and the other 52 patients were categorized as having non-fungal asthma without sensitization to either Aspergillus or Candida (the non-FA group). The proportion of males in the FA group was significantly higher than that in non-FA group (54\% vs. $31 \%, \mathrm{P}=0.0201)$. The patients with $\mathrm{FA}$ were significantly older than the patients with non-FA $($ mean \pm SD: $62.6 \pm 18.8$ vs. $49.9 \pm 17.4$ years old, $\mathrm{P}=0.0002$ ). The proportion of ACO tended to be higher in the FA group compared to the non-FA group, but the difference was not significant (16\% vs. $4 \%, \mathrm{P}=0.0541$ ). The peripheral blood eosinophil counts of the FA patients were significantly higher than those of the non-FA patients $($ mean \pm SD: $539.5 \pm 920.4 v s$. $161.4 \pm 213.2 / \mathrm{mm}^{3}, \mathrm{P}=0.0230$ ). The total $\mathrm{IgE}$ level of the FA patients was also significantly higher than that of the non-FA patients $($ mean \pm SD: $1,113 \pm 1,429$ vs. $279.1 \pm 313.2 \mathrm{IU} / \mathrm{mL}$, $\mathrm{P}<0.0001)$. Almost all of the patients with FA $(96 \%)$ were treated with an inhaled corticosteroid (ICS), and all of the non-FA patients were treated with an ICS. There was no significant difference in the proportion of patients treated with GINA Step5 therapy between the FA and non-FA groups ( $27 \%$ vs. $19 \%, \mathrm{P}=0.3723)$. The characteristics of the enrolled patients are summarized in Table 1.

\section{Frequencies of pulmonary emphysema on chest CT in allergic asthma with and without fungal sensitization}

The proportion of patients in the FA group who had pulmonary emphysema was significantly higher compared to the proportion in the non-FA group (18\% vs. $2 \%, \mathrm{P}=0.0040$ ). The proportion of patients who had bronchiectasis was also significantly higher among the patients with FA compared to the non-FA patients (48\% vs. $21 \%, \mathrm{P}=0.0046)$. In our comparison of the patients who were sensitized to Aspergillus and the non-FA patients, the frequency of pulmonary emphysema was significantly higher in the patients sensitized to Aspergillus compared to the non-FA patients $(14 \%$ vs. $2 \%, \mathrm{P}=0.0398)$. In the comparison of the patients who were sensitized to Candida and the non-FA patients, the frequency of pulmonary emphysema was also significantly higher in the patients sensitized to Candida compared to the non-FA group (17\% vs. 2\%, $\mathrm{P}=0.0198$ ) (Figure 2). The details of the patients with $\mathrm{FA}$ complicated with pulmonary emphysema are given in Table 2.

\section{Multivariate logistic regression analysis of risk factors associated with fungal sensitization}

We built a multivariate logistic regression model to assess the confounder effects of age, gender, packyears, and ACO in the relationship between pulmonary emphysema and fungal sensitization (Table 3). After adjustment for these variables, we found that the presence of pulmonary emphysema (OR 7.84, 95\% CI: 1.20-51.10) was independently associated with fungal sensitization. 
Table 1 The patients' characteristics

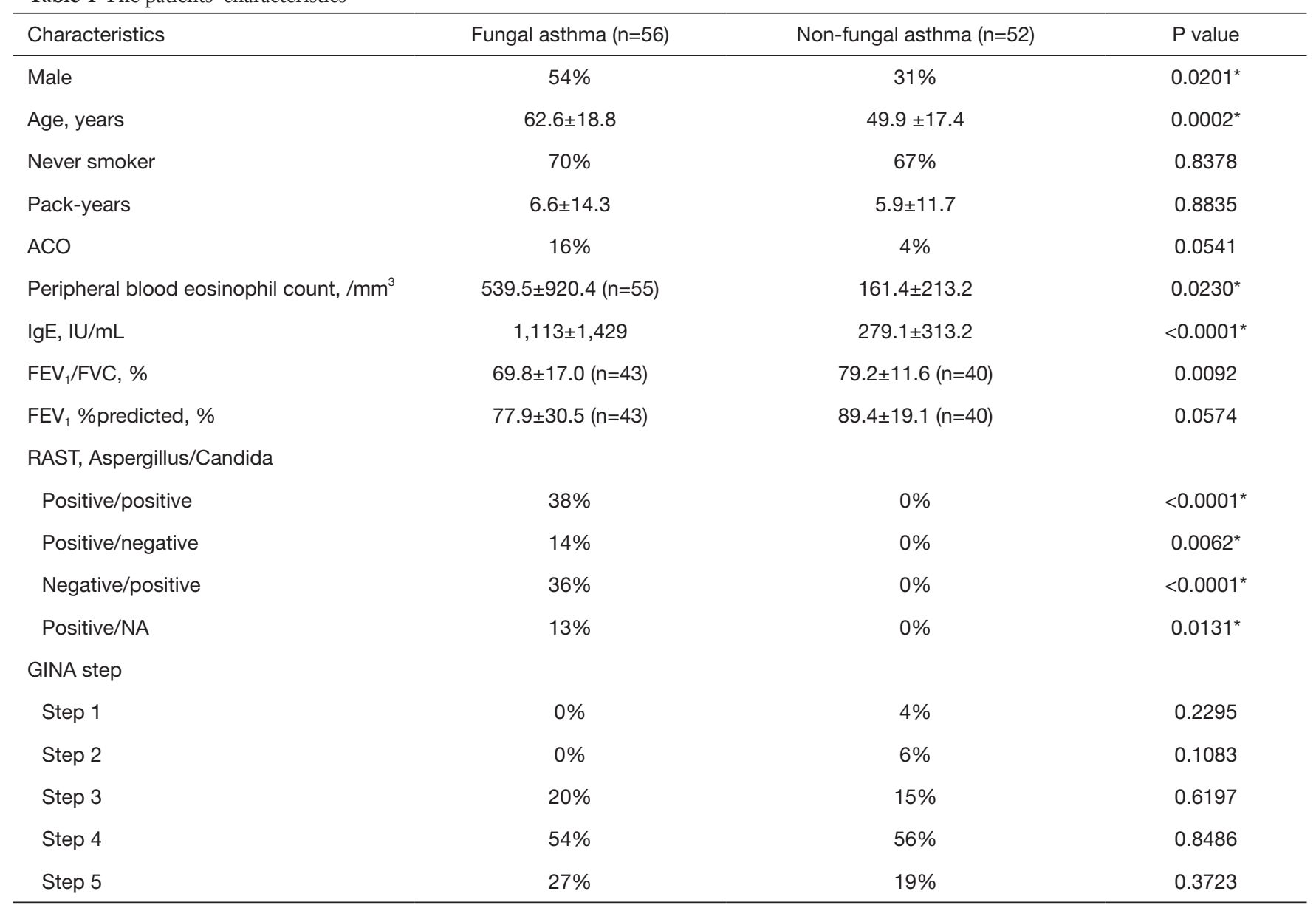

*, significant, $\mathrm{P}<0.05$. Data are presented as mean \pm SD when appropriate. ACO, asthma-COPD overlap; GINA, global initiative for asthma; NA, not available.

Higher patient age (OR 1.03, 95\% CI: 1.01-1.06) and lower pack-year value (OR 0.90, 95\% CI: 0.84-0.98) were also independently associated with fungal sensitization, indicating that the presence of pulmonary emphysema was the most relevant factor for the sensitization to Aspergillus and/or Candida.

\section{Discussion}

Our findings demonstrated that the frequency of pulmonary emphysema was higher in asthmatics who were sensitized to Aspergillus and/or Candida compared to asthmatics who were not sensitized to Aspergillus or Candida. We also observed that the presence of pulmonary emphysema was independently associated with sensitization to Aspergillus and/or Candida.

Several research groups have suggested that fungal colonization is associated with sensitization to Aspergillus. It was reported that in patients with asthma who were sensitized to Aspergillus, Aspergillus was frequently isolated from their sputa by culture and polymerase chain reaction (PCR) (11,12). Moreover, the basic etiology of allergic bronchopulmonary aspergillosis (ABPA) is thought to be allergic reaction to colonized Aspergillus (13). It is thus important for the diagnosis to check for Aspergillus in the patient's sputum. Antifungal therapy has been reported to be an effective treatment for ABPA $(14,15)$, suggesting a close relationship between the colonization and allergic reaction in Aspergillus.

In contrast to Aspergillus, the relationship between 
A

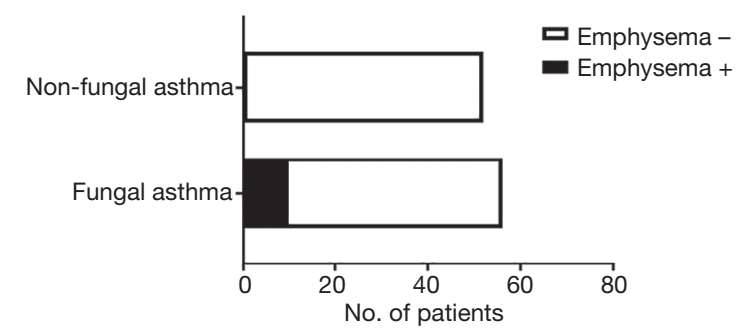

B

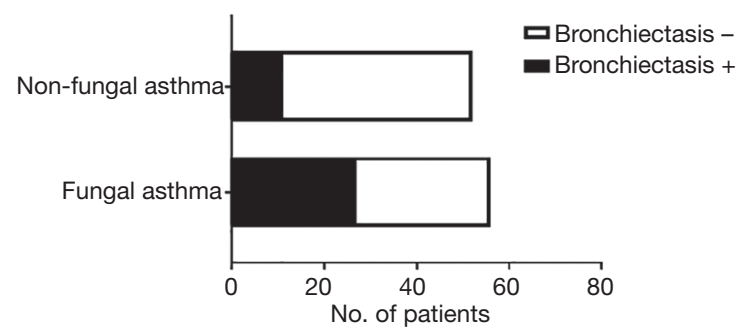

D

C
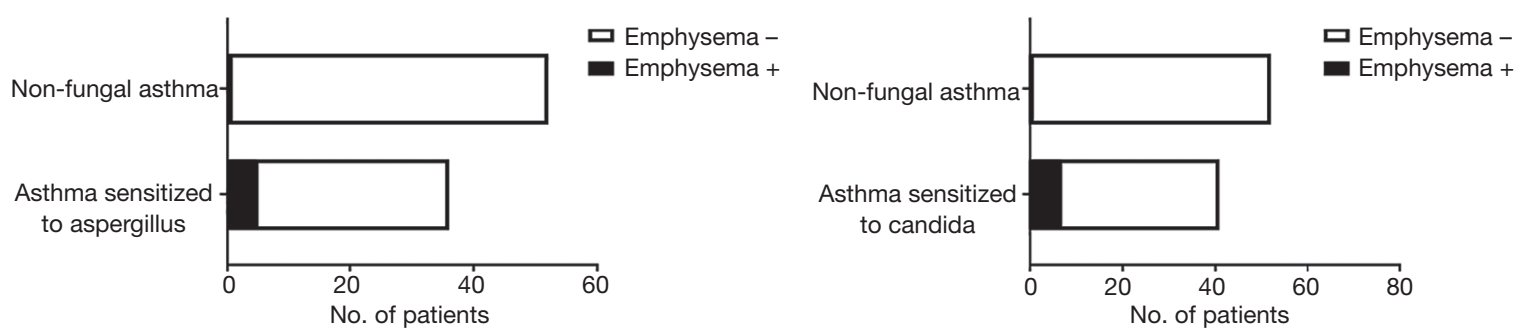

Figure 2 The frequencies of pulmonary emphysema and bronchiectasis on chest CT in asthma patients with and without fungal sensitization. (A) The frequencies of pulmonary emphysema on chest CT in asthma patients with and without fungal sensitization (18\% vs. $2 \%, \mathrm{P}=0.0040)$. (B) The frequencies of bronchiectasis on chest $\mathrm{CT}$ in asthma patients with and without fungal sensitization $(48 \% v s .21 \%$, $\mathrm{P}=0.0046$ ). (C) The frequencies of pulmonary emphysema on chest CT in the asthma patients who were sensitized to Aspergillus and the non-fungal asthma patients (14\% vs. $2 \%, \mathrm{P}=0.0398)$. (D) The frequencies of pulmonary emphysema on chest $\mathrm{CT}$ in the asthma patients who were sensitized to Candida and the non-fungal asthma patients (17\% vs. $2 \%, \mathrm{P}=0.0198)$.

sensitization and colonization in Candida is not well understood, because the evaluation of colonization in the lower respiratory tract is very difficult. Although Candida is not colonized in the human lower respiratory tract, it is frequently colonized in the oral cavity, and thus the isolation of Candida from sputum is common (16). However, in allergic bronchopulmonary mycosis (ABPM), Candida is the second most common causal species (after Aspergillus) (17), indicating that the colonization of Candida can cause an allergic reaction. The relationship between sensitization to and the colonization of Candida has also been studied in cystic fibrosis (CF), and although a relationship between the isolation of Candida from sputum and sensitization to Candida was not identified, the frequency of isolation from sputum and the rate of sensitization to Candida in patients with CF were high $(78 \%$ and $18 \%$, respectively) (18). In another report regarding CF and Candida, only patients who had at least one positive culture finding for Candida showed sensitization to Candida (19). These reports suggest that the colonization may be one of the causes of sensitization to Candida.
The precise mechanisms underlying an impaired local defense system in pulmonary emphysema are unknown. However, in COPD, the degree of emphysema was reported to be higher in patients who exhibited chronic colonization with Haemophilus influenzae compared to patients with no chronic colonization of H. influenza (20). In a study of hamsters with emphysema induced by porcine pancreatic elastase, chronic bacterial infection and colonization were reported to be highly correlated with lung emphysematous lesions (21). These reports suggest that pulmonary emphysema contributes to the disruption of the local defense system, leading to the colonization of microorganisms.

Considering these earlier findings, our present results suggest that a fungal colonization caused by disruption of the local defense system may lead to the fungal sensitization in pulmonary emphysema. However, regarding the association between pulmonary emphysema and colonization, another relationship is expected. Fungi are known to secrete several types of proteases including matrix metalloproteases (MMPs) (22), and epithelial cells 


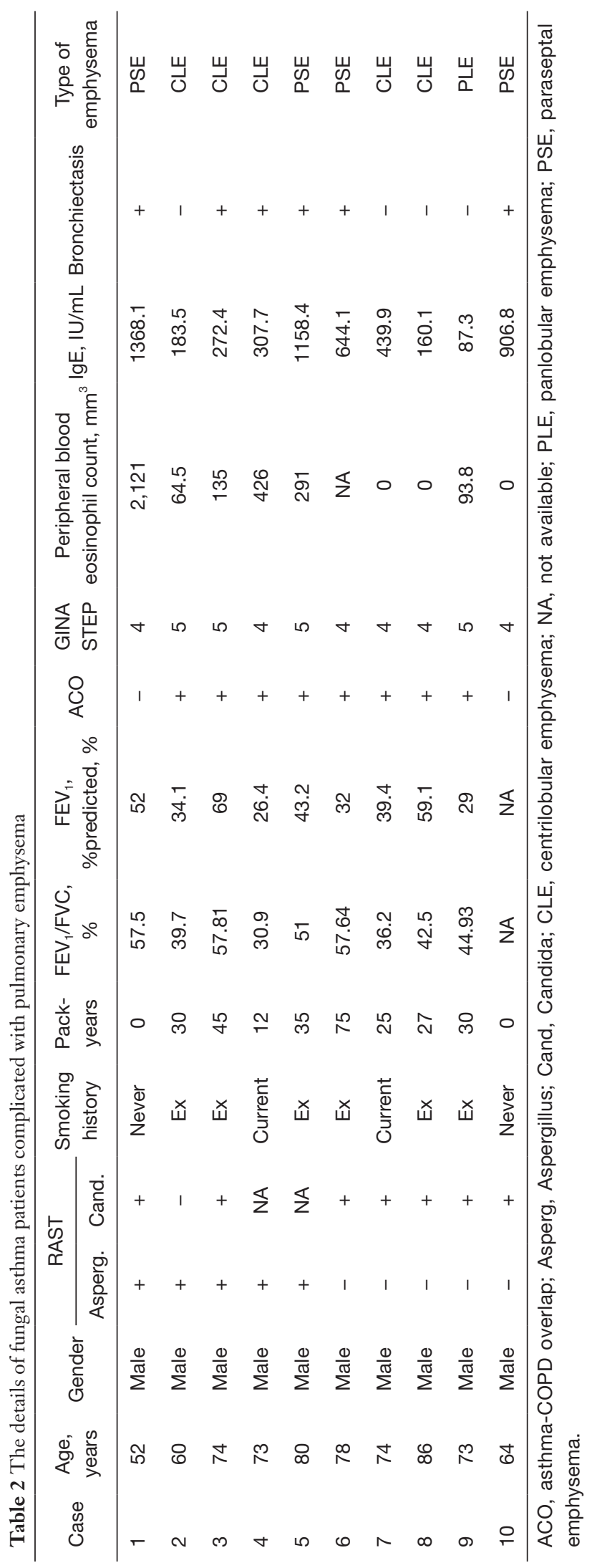

Table 3 Results from multivariate logistic regression model

\begin{tabular}{lll}
\hline Variables & OR $(95 \% \mathrm{Cl})$ & P value \\
\hline Pulmonary emphysema & $7.84(1.20-51.10)$ & $0.0313^{*}$ \\
Male & $2.24(0.83-6.06)$ & 0.1110 \\
Age & $1.03(1.01-1.06)$ & $0.0062^{*}$ \\
Pack year & $0.90(0.84-0.98)$ & $0.0092^{*}$ \\
ACO & $35.30(0.77-1620.00)$ & 0.0680
\end{tabular}

*, significant, $\mathrm{P}<0.05$. ACO, asthma-COPD overlap.

and inflammatory cells are known to secrete proteases in response to infection $(23,24)$. One of the major mechanisms in the formation of pulmonary emphysema is a proteaseantiprotease imbalance. Although it is not yet known whether the proteases related to fungus are involved in the formation of pulmonary emphysema, in mice with elastase-induced emphysema, an infection with Streptococcus pneumoniae resulted in increased MMP-12 production in the lungs and enhanced emphysema progression (24), suggesting that the production of proteases caused by infection can contribute to the development of pulmonary emphysema. In the present study, two of the ten FA patients with pulmonary emphysema were non-smokers and had bronchiectasis. Given the high prevalence of fungal colonization in bronchiectasis, a fungus might have contributed to the development of pulmonary emphysema in those two patients.

There are some study limitations to address. The first is that this study was a retrospective, single-center study with a small number of patients. A second limitation is that we did not evaluate fungal colonization, and thus the relationship between colonization and sensitization in our patient series is unknown. A third limitation is that our study population was not a general asthmatic population, because we enrolled only patients who had undergone chest CT. In general, chest CT is not performed in mild asthma. The ratio of severe asthma in this study is thus likely to be higher than that in the general asthmatic population. A fourth limitation is that there is a possibility that underdiagnosed patients with ABPA were included in this study.

\section{Conclusions}

Our findings demonstrated that fungal sensitization is associated with pulmonary emphysema. As a clinical implication, our results suggest that it is worthwhile to 
evaluate sensitization to Aspergillus and Candida if patients with asthma have pulmonary emphysema. In addition, given the previous reports regarding the association between fungal colonization and sensitization, our present findings also suggest that there are relationships among pulmonary emphysema and fungal colonization and sensitization. Further study is required to clarify these relationships in asthma.

\section{Acknowledgments}

Funding: None.

\section{Footnote}

Data Sharing Statement: Available at http://dx.doi. org/10.21037/jtd-20-995

Conflicts of Interest: All authors have completed the ICMJE uniform disclosure form (available at http://dx.doi. org/10.21037/jtd-20-995). All authors have no conflicts of interest to declare.

Ethical Statement: The authors are accountable for all aspects of the work in ensuring that questions related to the accuracy or integrity of any part of the work are appropriately investigated and resolved. This study was conducted under the approval of the Ethics Committee of The Fraternity Memorial Hospital and in accordance with the Declaration of Helsinki (as revised in 2013). Individual consent for this retrospective analysis was waived.

Open Access Statement: This is an Open Access article distributed in accordance with the Creative Commons Attribution-NonCommercial-NoDerivs 4.0 International License (CC BY-NC-ND 4.0), which permits the noncommercial replication and distribution of the article with the strict proviso that no changes or edits are made and the original work is properly cited (including links to both the formal publication through the relevant DOI and the license). See: https://creativecommons.org/licenses/by-nc-nd/4.0/.

\section{References}

1. Black PN, Udy AA, Brodie SM. Sensitivity to fungal allergens is a risk factor for life-threatening asthma. Allergy 2000;55:501-4.

2. Zureik M, Neukirch C, Leynaert B, et al. Sensitisation to airborne moulds and severity of asthma: cross sectional study from European Community respiratory health survey. BMJ 2002;325:411-4.

3. Denning DW, O'Driscoll BR, Hogaboam CM, et al. The link between fungi and severe asthma: a summary of the evidence. Eur Respir J 2006;27:615-26.

4. Masaki K, Fukunaga K, Matsusaka M, et al. Characteristics of severe asthma with fungal sensitization. Ann Allergy Asthma Immunol 2017;119:253-7.

5. Kennedy JL, Heymann PW, Platts-Mills TA. The role of allergy in severe asthma. Clin Exp Allergy 2012;42:659-69.

6. Woolnough KF, Richardson M, Newby C, et al. The relationship between biomarkers of fungal allergy and lung damage in asthma. Clin Exp Allergy 2017;47:48-56.

7. Máiz L, Nieto R, Cantón R, et al. Fungi in Bronchiectasis: A Concise Review. Int J Mol Sci 2018;19:142.

8. Denning DW, Riniotis K, Dobrashian R, et al. Chronic cavitary and fibrosing pulmonary and pleural aspergillosis: case series, proposed nomenclature change, and review. Clin Infect Dis 2003;37 Suppl 3:S265-80.

9. Smith NL, Denning DW. Underlying conditions in chronic pulmonary aspergillosis including simple aspergilloma. Eur Respir J 2011;37:865-72.

10. Trudzinski FC, Seiler F, Wilkens H, et al. Microbiological airway colonization in COPD patients with severe emphysema undergoing endoscopic lung volume reduction. Int J Chron Obstruct Pulmon Dis 2018;13:29-35.

11. Farrant J, Brice H, Fowler S, et al. Fungal sensitisation in severe asthma is associated with the identification of Aspergillus fumigatus in sputum. J Asthma 2016;53:732-5.

12. Fairs A, Agbetile J, Hargadon B, et al. IgE sensitization to Aspergillus fumigatus is associated with reduced lung function in asthma. Am J Respir Crit Care Med 2010;182:1362-8.

13. Gago S, Denning DW, Bowyer P. Pathophysiological aspects of Aspergillus colonization in disease. Med Mycol 2019;57:S219-27.

14. Wark PA, Hensley MJ, Saltos N, et al. Anti-inflammatory effect of itraconazole in stable allergic bronchopulmonary aspergillosis: a randomized controlled trial. J Allergy Clin Immunol 2003;111:952-7.

15. Stevens DA, Schwartz HJ, Lee JY, et al. A randomized trial of itraconazole in allergic bronchopulmonary aspergillosis. N Engl J Med 2000;342:756-62.

16. Pendleton KM, Huffnagle GB, Dickson RP. The significance of Candida in the human respiratory tract: our evolving understanding. Pathog Dis 2017;75:ftx029.

17. Chowdhary A, Agarwal K, Kathuria S, et al. Allergic 
bronchopulmonary mycosis due to fungi other than Aspergillus: a global overview. Crit Rev Microbiol 2014;40:30-48.

18. Baxter CG, Moore CB, Jones AM, et al. IgE-mediated immune responses and airway detection of Aspergillus and Candida in adult cystic fibrosis. Chest 2013;143:1351-7.

19. Máiz L, Cuevas M, Quirce S, et al. Serologic IgE immune responses against Aspergillus fumigatus and Candida albicans in patients with cystic fibrosis. Chest 2002;121:782-8.

20. Tufvesson E, Markstad H, Bozovic G, et al. Inflammation and chronic colonization of Haemophilus influenzae in sputum in COPD patients related to the degree of emphysema and bronchiectasis in high-resolution computed tomography. Int J Chron Obstruct Pulmon Dis
2017;12:3211-9.

21. Wang D, Wang Y, Liu YN. Experimental pulmonary infection and colonization of Haemophilus influenzae in emphysematous hamsters. Pulm Pharmacol Ther 2010;23:292-9.

22. Monod M, Capoccia S, Lechenne B, et al. Secreted proteases from pathogenic fungi. Int J Med Microbiol 2002;292:405-19.

23. Chiang TY, Tsao SM, Yeh CB, et al. Matrix metalloproteinases in pneumonia. Clin Chim Acta. 2014;433:272-7.

24. Takahashi S, Ishii M, Namkoong H, et al. Pneumococcal Infection Aggravates Elastase-Induced Emphysema via Matrix Metalloproteinase 12 Overexpression. J Infect Dis 2016;213:1018-30.
Cite this article as: Kono $\mathrm{Y}$, To M, Tsuzuki R, Yamawaki S, Soeda S, To Y. Pulmonary emphysema is associated with fungal sensitization in asthma. J Thorac Dis 2020;12(10):5879-5886. doi: 10.21037/jtd-20-995 